\title{
Smoothness and geometry of boundaries associated to skeletal structures, II: Geometry in the Blum case
}

\author{
James Damon
}

\begin{abstract}
A skeletal structure $(M, U)$ in $\mathbb{R}^{n+1}$ is a special type of $n$-dimensional Whitney stratified set $M$ on which is defined a multivalued 'radial vector field' $U$. This is an extension of the notion of the Blum medial axis of a region in $\mathbb{R}^{n+1}$ with generic smooth boundary. For such a skeletal structure an 'associated boundary' $\mathcal{B}$ is defined. In part I of this paper, we introduced radial and edge shape operators, which are geometric invariants of the radial vector field $U$ on $M$, and a 'radial flow' from $M$ to $\mathcal{B}$. In this paper, in the partial Blum case we derive formulas for the differential geometric shape operator of the boundary (and hence all curvature invariants) in terms of the shape operators on the medial axis. We further derive the effects of a diffeomorphism of the skeletal structure on the radial and edge shape operators using a distortion operator which is computed from the second derivative of the diffeomorphism evaluated on the unit radial vector field. This allows one to compute the geometry of the boundary associated to a deformed skeletal structure purely in terms of operators defined on the original skeletal set.
\end{abstract}

\section{Introduction}

In the first part of this paper [Dam03], we introduced the notion of a skeletal structure $(M, U)$ as a generalization of the Blum medial axis $M$ of a region with smooth boundary. It consists of a Whitney stratified set $M$ together with the multivalued radial vector field $U$ from points of $M$ to the corresponding points of tangency on the boundary, e.g. Figure 1.

We may write $U=r \cdot U_{1}$ for a unit vector field $U_{1}$ and radius function $r$. For such skeletal structures we introduced a radial shape operator $S_{\mathrm{rad}}$, an edge shape operator $S_{\mathrm{E}}$ and a compatibility 1 -form $\eta_{U}$. Associated to $S_{\text {rad }}$ are the 'principal radial curvatures' $\kappa_{\mathrm{r} i}$, the eigenvalues of $S_{\mathrm{rad}}$, and to $S_{\mathrm{E}}$ the 'principal edge curvatures' $\kappa_{\mathrm{E} i}$, which are generalized eigenvalues of $S_{\mathrm{E}}$.

Using these objects we gave a trio of conditions, radial curvature condition, edge condition, and compatibility condition, which are (necessary and) sufficient to ensure that the 'associated boundary' $\mathcal{B}$ is smooth. The skeletal set $M$ and associated boundary $\mathcal{B}$ are naturally related by a radial map defined using the radial vector field $U$. The radial map is the time-one map of an associated 'radial flow' from $M$ to $\mathcal{B}$. The three conditions control the properties of the radial flow.

In this second part we investigate the geometry of the associated boundary $\mathcal{B}$ in the 'partial Blum case'. This requires only one of the conditions which $M$ must satisfy to be a Blum medial axis; namely, the radial vector is orthogonal to the boundary, which is guaranteed by a 'compatibility

Received 23 October 2002, accepted in final form 15 August 2003, published online 15 October 2004. 2000 Mathematics Subject Classification 53A07, 58A35 (primary), 68U05, 57N80 (secondary).

Keywords: skeletal structures, Whitney stratified sets, Blum medial axis, radial shape operator, radial flow, distortion operator.

Partially supported by National Science Foundation Grant DMS-0103862.

This journal is (C) Foundation Compositio Mathematica 2004. 


\section{J. DAMON}

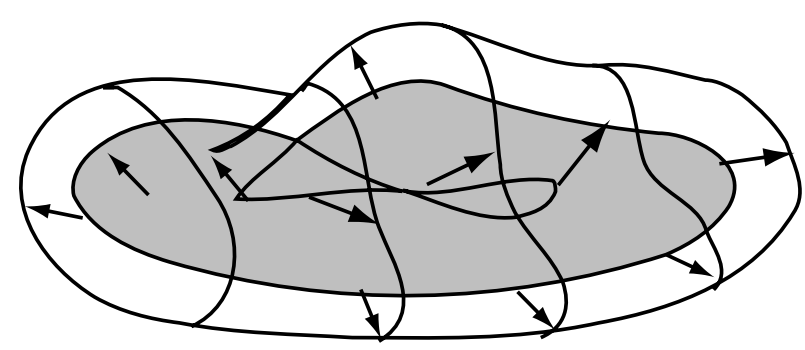

Figure 1. Blum medial axis giving a skeletal structure of an object.

condition'. By contrast, the full Blum condition also requires additional conditions such as, for example, at smooth points of $M$ the two values of $U$ must have the same length and their difference must be orthogonal to $M$.

In the partial Blum case, if the radius function is constant on an open set of $M$, then it follows that $U$ is orthogonal to $M$. Thus, the boundary is a parallel manifold whose geometry is determined in a standard way from $M$ (see e.g. [Spi75, vol. III, ch. 3]). When $r$ is not constant (and $U$ is no longer orthogonal to $M$ ), there are more complicated relations between the differential geometry of the boundary and that of the medial axis. These relations also involve derivative properties of the radius function $r$. The 2D case for boundary curves originated with [BN78]. For boundary surfaces in 3D, Nackman and Pizer obtained formulas for the Gaussian and mean curvatures of the boundary [Nac82, NP85]. In the opposite direction, differential geometric properties of the medial axis have been obtained from the differential geometry of the boundary by Siersma, Sotomayor, and Garcia [Sie99, SSG99] (and also see [VaM03a, VaM03b]). Actually in both of these cases the relationship is with the differential geometry of a parallel surface of the boundary.

We take a different approach to this problem which directly considers the 'geometry of the radial vector field' as expressed by the radial and edge shape operators. To understand the geometry of the boundary we determine how the radial shape operator for level hypersurfaces $\mathcal{B}_{t}$ evolves under the radial flow, obtaining an expression in terms of the initial radial shape operator $S_{\text {rad }}$ (and the edge shape operator for points corresponding to the edge of $M$ ). This allows us to determine explicitly the differential geometry of the boundary $\mathcal{B}$. When the compatibility condition holds on an open subset $W$ of smooth points of $M$, the lines along $U$ from this set intersect $\mathcal{B}$ orthogonally so we are partially in the Blum situation. In this case, we explicitly express (in Theorem 3.2) the differential geometric shape operator $S_{\mathcal{B}}$ of $\mathcal{B}$ on the subset corresponding to $W$ by

$$
S_{\mathcal{B} \mathbf{v}^{\prime}}=\left(I-r S_{\mathbf{v}}\right)^{-1} S_{\mathbf{v}}
$$

where $S_{\mathbf{v}}$ denotes a matrix representation of $S_{\text {rad }}$ with respect to a basis $\mathbf{v}$, and $S_{\mathcal{B} \mathbf{v}^{\prime}}$ is a matrix representation with respect to an associated basis $\mathbf{v}^{\prime}$. From this we deduce formulas for the principal curvatures $\kappa_{i}$ of $\mathcal{B}$ in terms of the principal radial curvatures $\kappa_{\mathrm{r} i}$ and conversely

$$
\kappa_{i}=\frac{\kappa_{\mathrm{r} i}}{\left(1-r \cdot \kappa_{\mathrm{r} i}\right)} \quad \text { or } \quad \kappa_{\mathrm{r} i}=\frac{\kappa_{i}}{\left(1+r \cdot \kappa_{i}\right)}
$$

(in particular, although $S_{\text {rad }}$ is not self-adjoint, it can be diagonalized with real eigenvalues). In addition, we show that the radial flow sends the eigendirections of $S_{\text {rad }}$ to the principal normal directions of $\mathcal{B}$.

In the case of 'crest points' on the boundary, which correspond to edge points of the medial axis (see [BGT96] or [Gib00]), we obtain an analogous result expressing the differential geometric shape operator of $\mathcal{B}$ at a crest point in terms of the edge shape operator $S_{\mathrm{E} \mathbf{v}}$ by $S_{\mathcal{B} \mathbf{v}^{\prime}}=\left(I_{n-1,1}-r S_{\mathrm{E} \mathbf{v}}\right)^{-1} S_{\mathrm{E} \mathbf{v}}$, where $I_{n-1,1}$ is obtained from the identity matrix by replacing the 


\section{GEOMETRY OF BOUNDARIES}

last element by 0 . However, there is not such a simple relationship between the principal curvatures of $\mathcal{B}$ and the principal edge curvatures.

We then determine the relation between the differential geometry of $\mathcal{B}$ and that of the medial axis $M$ via $S_{\text {rad }}$. In Proposition 4.1 we give a relation between $S_{\text {rad }}$ and $S_{\text {med }}$, the differential geometric shape operator of $M$. This relation also involves a 'radial Hessian operator' $H_{\mathrm{r}}$ as well as an additional non-self-adjoint operator $Z$. In light of the formulas we obtain in terms of $S_{\mathbf{v}}$, this explains the added complications in computing the differential geometric shape operator $S_{\mathcal{B}}$ of the boundary in terms of $S_{\text {med }}$.

Second, we determine in $\S 5$ the effect of a 'deforming diffeomorphism' $\varphi$ of (an arbitrarily small neighborhood of) the skeletal structure $(M, U)$ to yield a skeletal structure $\left(M^{\prime}, V\right)=$ $(\varphi(M), d \varphi(U))$. We introduce a radial distortion operator $Q_{\varphi \mathbf{v}}$ and edge distortion operator $Q_{\mathrm{E} \varphi, \mathbf{v}}$ defined in terms of the second derivative of $\varphi$ at points on $M$. A third operator $E_{\varphi \mathrm{v}}$ measures the failure of $\varphi$ to preserve orthogonality to the skeletal set at edge points. Then, we measure the change in the radial and edge shape operators as a result of applying $\varphi$. With respect to a basis $\mathbf{v}$ for $T_{x_{0}} M$ with associated basis $\mathbf{v}^{\prime}$ in the image, the image shape operators are given by Theorem 5.4

$$
S_{\mathbf{v}^{\prime}}=\sigma\left(S_{\mathbf{v}}+Q_{\varphi, \mathbf{v}}\right) \quad \text { and } \quad S_{\mathrm{E} \mathbf{v}^{\prime}}=\sigma\left(S_{\mathrm{E} \mathbf{v}}+Q_{\mathrm{E} \varphi, \mathbf{v}}+E_{\varphi \mathbf{v}}\right),
$$

where $\sigma$ is a 'radial scaling factor' relating $\|U\|$ and $\|V\|$.

Hence, these distortion operators allow us to determine whether the radial curvature and edge conditions for smoothness of the associated boundary of the image $\left(M^{\prime}, V\right)$ continue to hold. Also, for diffeomorphisms which satisfy a radial rigidity condition we show that the compatibility 1-form pushes forward to be the compatibility 1-form of the image skeletal structure $(\varphi(M), d \varphi(U))$. This implies that the compatibility condition is unaltered by such a diffeomorphism. Thus, we deduce sufficient conditions that applying a diffeomorphism to a skeletal structure will yield a skeletal structure which still satisfies the three conditions for the smoothness of the associated boundary (Theorem 5.7).

Moreover, in light of the above results, we are able to determine, in terms of the initial radial and edge shape operators and the distortion operators on the initial Blum medial axis, the changes in the differential geometric properties of the associated boundary for the image. What is rather surprising is how the changes in differential geometric properties under diffeomorphisms can be reduced to a 'linear relation' combined with the specific non-linear relation between radial shape operators and geometric shape operators for the boundary.

\section{Preliminaries: shape operators and radial flow}

We recall from part I [Dam03] that, beginning with a skeletal structure $(M, U)$ in $\mathbb{R}^{n+1}$, we associate a boundary $\mathcal{B}=\{x+U(x): x \in M$, all values of $U\}$. A standard example we consider will be the Blum medial axis $M$ of a region $\Omega$ with generic smooth boundary $\mathcal{B}$ and its associated (multivalued) radial vector field $U$. Then, the associated boundary $\mathcal{B}$ we consider here will be the initial boundary of the object. We relate the boundary and skeletal set via the radial flow and the radial map which is the time-one map of the radial flow. To define these globally requires the introduction of the 'double of $M^{\prime}$ '; however, in the neighborhood $W$ of a point $x_{0} \in M$ with a smooth single-valued choice for $U$, we define a local representation of the radial flow by $\psi_{t}(x)=x+t \cdot U(x)$, and the radial map $\psi_{1}(x)=x+U(x)$.

We define 'shape operators' for the skeletal structure $(M, U)$. At a smooth point $x_{0}$ of $M$ we choose a 'smooth value' of $U$. We recall that in a neighborhood of any smooth point of $M$, values of $U$ on one side form a smooth vector field. By a smooth value of $U$ we mean such a smooth choice of $U$ values. Then, $U=r \cdot U_{1}$ for a unit radial vector field $U_{1}$. 


\section{J. DAMON}

Radial shape operator. For $v \in T_{x_{0}} M$, we define

$$
S_{\mathrm{rad}}(v)=-\operatorname{proj}_{U}\left(\frac{\partial U_{1}}{\partial v}\right)
$$

where $\operatorname{proj}_{U}$ denotes projection onto $T_{x_{0}} M$ along $U$ (in general, this is not orthogonal projection). $S_{\mathbf{v}}$ denotes the matrix representation of $S_{\text {rad }}$ with respect to a basis $\mathbf{v}=\left\{v_{1}, \ldots, v_{n}\right\}$. The principal radial curvatures $\kappa_{\mathrm{r} i}$ are the eigenvalues of $S_{\mathrm{rad}}$.

For a non-edge point $x_{0} \in M$, a value of $U$ extends to be smooth on some local neighborhood component $M_{\alpha}$ of $x_{0}$. For this smooth value of $U$, we may likewise define the radial shape operator at $x_{0}$.

Remark. Thus, the radial shape operator is also multivalued in that at a non-edge point $x_{0}$ there will be a radial shape operator for each value of $U$ at $x_{0}$, which at smooth points means a value for each side of $M$.

We may write the derivatives $\partial U_{1} / \partial v_{i}$ in vector notation (see $(2.2)$ of [Dam03])

$$
\frac{\partial U_{1}}{\partial \mathbf{v}}=A_{\mathbf{v}} \cdot U_{1}-S_{\mathbf{v}}^{\mathrm{T}} \cdot \mathbf{v}
$$

where $\partial U_{1} / \partial \mathbf{v}$ is a column vector with vector entries $\partial U_{1} / \partial v_{i}$, and $A_{\mathbf{v}} \cdot U_{1}$ denotes the column vector with entries $a_{i} \cdot U_{1}$. We abuse notation to let $\mathbf{v}$ also denote a column vector with $i$ th entry the vector $v_{i}$. Using this we compute the derivative of the radial flow (see (4.2) of [Dam03]):

$$
\frac{\partial \psi_{t}}{\partial \mathbf{v}}=t\left(d r(\mathbf{v})+r \cdot A_{\mathbf{v}}\right) \cdot U_{1}+\left(I-t r \cdot S_{\mathbf{v}}\right)^{\mathrm{T}} \cdot \mathbf{v}
$$

where $\partial \psi_{t} / \partial \mathbf{v}$ and $d r(\mathbf{v})$ are column vectors with $i$ th entries $\partial \psi_{t} / \partial v_{i}$, respectively $d r\left(v_{i}\right)$.

Edge shape operator. For an edge point $x_{0}$, with a smooth value of $U$ defined on a neighborhood of $x_{0}$ (corresponding to one side of $M$ ), and a normal vector field $\mathbf{n}$ to $M$, we define

$$
S_{\mathrm{E}}(v)=-\operatorname{proj}^{\prime}\left(\frac{\partial U_{1}}{\partial v}\right) .
$$

Here proj' denotes projection onto $T_{x_{0}} \partial M \oplus\langle\mathbf{n}\rangle$ along $U$ (again this is not orthogonal). Let $\mathbf{v}=$ $\left\{v_{1}, \ldots, v_{n}\right\}$ be a basis of $T_{x_{0}} M$ so that $\left\{v_{1}, \ldots, v_{n-1}\right\}$ is a basis of $T_{x_{0}} \partial M$, and $v_{n}$ maps under the edge parametrization map to $c \cdot U$ for $c \geqslant 0$. We refer to $\mathbf{v}$ as a special basis for $T_{x_{0}} M$. Then, $S_{\mathrm{E} \mathbf{v}}$ is a matrix representation of $S_{\mathrm{E}}$ with respect to the basis $\mathbf{v}$ in the source and $\left\{v_{1}, \ldots, v_{n-1}, \mathbf{n}\right\}$ in the target. Again by (2.4) of [Dam03]

$$
\frac{\partial U_{1}}{\partial \mathbf{v}}=A_{U} \cdot U_{1}-S_{\mathrm{E} \mathbf{v}}^{\mathrm{T}} \cdot\left(\begin{array}{c}
\tilde{\mathbf{v}} \\
\mathbf{n}
\end{array}\right)
$$

where $\tilde{\mathbf{v}}$ is the $(n-1)$-dimensional column vector with $i$ th entry the vector $v_{i}$. Then, the derivative of the radial flow is given by the following (see (4.10) of [Dam03]):

$$
\frac{\partial \psi_{t}}{\partial \mathbf{v}}=\tilde{A}_{U} \cdot U_{1}+\left(I_{n-1,1}-t r \cdot S_{\mathrm{E} \mathbf{v}}\right)^{\mathrm{T}} \cdot\left(\begin{array}{c}
\tilde{\mathbf{v}} \\
\mathbf{n}
\end{array}\right) .
$$

Here $I_{n-1,1}$ is the $n \times n$ diagonal matrix with 1 in the first $n-1$ positions and 0 otherwise. The principal edge curvatures $\kappa_{\mathrm{E} i}$ are the generalized eigenvalues of $\left(S_{\mathrm{E} \mathbf{v}}, I_{n-1,1}\right)$ (i.e. $\lambda$ such that $S_{\mathrm{Ev}}-\lambda \cdot I_{n-1,1}$ is singular). 


\section{GEOMETRY OF BOUNDARIES}

\section{Evolution of the shape operators under the radial flow}

\section{Evolution of the radial shape operator from smooth points}

We first consider the evolution of the radial shape operator under the radial flow. Let $x_{0} \in M_{\text {reg }}$, and let $\left\{v_{1}, \ldots, v_{n}\right\}$ be a basis for $T_{x_{0}} M$. We suppose we have chosen a smooth value of $U$ in a neighborhood of $x_{0}$. If $x_{0}$ is a non-edge singular point, then we can carry out an analogous argument on a local component for $x_{0}$.

For a given $t$, let

$$
v_{i}^{\prime}=d \psi_{t}\left(v_{i}\right)=\frac{\partial \psi_{t}}{\partial v_{i}} \quad \text { for } i=1, \ldots, n .
$$

We suppose that $1 /(t r)$ is not an eigenvalue of $S_{\text {rad }}\left(\right.$ at $\left.x_{0}\right)$. Then, by the proof of Proposition 4.1 of [Dam03], $\psi_{t}$ maps a neighborhood $W$ of $x_{0}$ diffeomorphically to a smooth submanifold transverse to $U\left(x_{0}\right)$. Thus, the image of $U$ along $\psi_{t}$ remains transverse in some neighborhood of $x_{0}^{\prime}=\psi_{t}\left(x_{0}\right)$ to $W^{\prime}=\psi_{t}(W) \subset \mathcal{B}_{t}$. Hence, it has a well-defined radial shape operator, which we denote by $S_{\operatorname{rad} t}$. We will compute $S_{\mathbf{v}^{\prime} t}$, the matrix representation of $S_{\operatorname{rad} t}$ with respect to the basis $\left\{v_{i}^{\prime}\right\}$.

Proposition 2.1. Suppose that, at a smooth point $x_{0} \in M_{\mathrm{reg}}$, we have a smooth value of $U$ and a basis $\left\{v_{i}\right\}$ for $T_{x_{0}} M$. Let $\left\{v_{i}^{\prime}\right\}$ denote the image of $\left\{v_{i}\right\}$ under $d \psi_{t}\left(x_{0}\right)$. If $1 /(t r)$ is not an eigenvalue of the radial shape operator $S_{\mathbf{v}}$ at $x_{0}$, then the radial shape operator $S_{\mathbf{v}^{\prime} t}$ for $\mathcal{B}_{t}$ at $x_{0}^{\prime}=\psi_{t}\left(x_{0}\right)$ for the corresponding smooth value of $U$ is given by

$$
S_{\mathbf{v}^{\prime} t}=\left(I-t r \cdot S_{\mathbf{v}}\right)^{-1} S_{\mathbf{v}} .
$$

Proof. To carry out the computation we again use vector notation and let $\mathbf{v}^{\prime}$ denote the column vector with $i$ th entry the vector $v_{i}^{\prime}$. We wish to compute $\partial U_{1} / \partial v_{i}^{\prime}$.

We first claim $\partial U_{1} / \partial v_{i}^{\prime}=\partial U_{1} / \partial v_{i}$. To see this, we let $\gamma(u)$ be a curve in $W$ with $\gamma(0)=x_{0}$ and derivative $\gamma^{\prime}(0)=v_{i}$. Then, $\gamma_{1}(u)=\psi_{t} \circ \gamma(u)$ is a curve in $W^{\prime} \subset \mathcal{B}_{t}$ with $\gamma_{1}(0)=x_{0}^{\prime}$ and derivative $\gamma_{1}^{\prime}(0)=v_{i}^{\prime}$. Then, note that one way to compute is

$$
\frac{\partial U_{1}}{\partial v_{i}^{\prime}}=\left.\frac{\partial\left(U_{1} \circ \gamma_{1}\right)(u)}{\partial u}\right|_{u=0}=\left.\frac{\partial\left(U_{1} \circ \gamma\right)(u)}{\partial u}\right|_{u=0},
$$

since $U_{1}$ at $\gamma_{1}(u)$ is just the translation of $U_{1}$ at $\gamma(u)$, or

$$
\frac{\partial U_{1}}{\partial v_{i}^{\prime}}=\frac{\partial U_{1}}{\partial v_{i}}
$$

Thus, in vector form, $\partial U_{1} / \partial \mathbf{v}^{\prime}=\partial U_{1} / \partial \mathbf{v}$. Then, first from (1.2),

$$
\frac{\partial U_{1}}{\partial \mathbf{v}}=A_{\mathbf{v}} \cdot U_{1}-S_{\mathbf{v}}^{\mathrm{T}} \cdot \mathbf{v} .
$$

Also, from the calculation of the derivative of the radial flow (1.2),

$$
\mathbf{v}^{\prime}=\frac{\partial \psi_{t}}{\partial \mathbf{v}}=t\left(d r(\mathbf{v})+r \cdot A_{\mathbf{v}}\right) \cdot U_{1}+\left(I-t r \cdot S_{\mathbf{v}}\right)^{\mathrm{T}} \mathbf{v} .
$$

Hence, provided $1 /(t r)$ is not an eigenvalue of $S_{\mathbf{v}},\left(I-t r \cdot S_{\mathbf{v}}\right)^{\mathrm{T}}$ is invertible, so we may solve for $\mathbf{v}$ and substitute into (2.3), using that $\partial U_{1} / \partial \mathbf{v}^{\prime}=\partial U_{1} / \partial \mathbf{v}$ to obtain

$$
\frac{\partial U_{1}}{\partial \mathbf{v}^{\prime}}=A_{\mathbf{v}^{\prime}}^{\prime} \cdot U_{1}-\left(I-t r \cdot S_{\mathbf{v}}^{\mathrm{T}}\right)^{-1} S_{\mathbf{v}}^{\mathrm{T}} \mathbf{v}^{\prime},
$$

where

$$
A_{\mathbf{v}^{\prime}}^{\prime}=A_{\mathbf{v}}-t \cdot\left(I-t r \cdot S_{\mathbf{v}}^{\mathrm{T}}\right)^{-1} S_{\mathbf{v}}^{\mathrm{T}}\left(d r(\mathbf{v})+r \cdot A_{\mathbf{v}}\right) .
$$




\section{J. DAMON}

Note that $S_{\mathbf{v}}$ and $\left(I-t r \cdot S_{\mathbf{v}}\right)^{-1}$ commute so they may be written in either order. By the definition of $S_{\operatorname{rad} t}$ and (2.5) we obtain

which implies (2.1).

$$
S_{\mathbf{v}^{\prime} t}^{\mathrm{T}}=\left(I-t r \cdot S_{\mathbf{v}}^{\mathrm{T}}\right)^{-1} S_{\mathbf{v}}^{\mathrm{T}}
$$

\section{Principal radial curvatures for $\mathcal{B}_{t}$}

Now, we can deduce information about the principal radial curvatures at $x_{0}^{\prime}$ in terms of those at $x_{0}$.

Corollary 2.2. Under the assumptions of Proposition 2.1, there is a correspondence (counting multiplicities) between the principal radial curvatures $\kappa_{\mathrm{r} i}$ of $M$ at $x_{0}$ and $\kappa_{\mathrm{r} t i}$ of $\mathcal{B}_{t}$ at $x_{0}^{\prime}$ given by

$$
\kappa_{\mathrm{r} t i}=\frac{\kappa_{\mathrm{r} i}}{\left(1-t r \kappa_{\mathrm{r} i}\right)} \quad \text { or equivalently } \quad \kappa_{\mathrm{r} i}=\frac{\kappa_{\mathrm{r} t i}}{\left(1+t r \kappa_{\mathrm{r} t i}\right)} .
$$

Furthermore, if $e_{i}$ is an eigenvector for the eigenvalue $\kappa_{\mathrm{r} i}$, then $e_{i}^{\prime}$, which has the same coordinates with respect to $\mathbf{v}^{\prime}$ as $e_{i}$ has with respect to $\mathbf{v}$, is an eigenvector with eigenvalue $\kappa_{\mathrm{r}} t i$.

Proof. We first use (2.1) to solve for $S_{\mathbf{v}}$ in terms of $S_{\mathbf{v}^{\prime} t}$. From (2.1) we may write

$$
S_{\mathbf{v}^{\prime} t}=S_{\mathbf{v}}\left(I+t r \cdot S_{\mathbf{v}^{\prime} t}\right) \text {. }
$$

Then, $\left(I+t r \cdot S_{\mathbf{v}^{\prime} t}\right)$ is also non-singular, for if there were a vector $v$ that $\left(I+t r \cdot S_{\mathbf{v}^{\prime} t}\right)(v)=0$, then, by (2.6), $S_{\mathbf{v}^{\prime} t}(v)=0$; and hence, $v=I(v)=0$. Thus, we may use (2.6) to solve for $S_{\mathbf{v}}$,

$$
S_{\mathbf{v}}=\left(I+t r \cdot S_{\mathbf{v}^{\prime} t}\right)^{-1} S_{\mathbf{v}^{\prime} t} .
$$

Now, (2.1) and (2.7) allow us to compare the eigenvalues of $S_{\mathbf{v}}$ and $S_{\mathbf{v}^{\prime} t}$, and thus the principal radial curvatures. If $\kappa_{\mathrm{r} i}$ is an eigenvalue for $S_{\mathbf{v}}$ with eigenvector $e_{i}$, then $\left(1-t r \cdot \kappa_{\mathrm{r} i}\right)^{-1}$ is an eigenvalue for $\left(I-t r \cdot S_{\mathbf{v}}\right)^{-1}$ with the same eigenvector; and hence, by $(2.1), \kappa_{\mathrm{r} i} \cdot\left(1-t r \kappa_{\mathrm{r} i}\right)^{-1}$ is an eigenvalue of $S_{\mathbf{v}^{\prime} t}$ with eigenvector $e_{i}$. We can carry out a similar argument interchanging the roles of $S_{\mathbf{v}}$ and $S_{\mathbf{v}^{\prime} t}$ using instead (2.7). Hence, we obtain the correspondence between the principal curvatures and principal radial curvatures as well as the corresponding eigenvectors.

\section{Evolution of the radial shape operator from edge points}

We can carry out an analogous line of reasoning for the evolution of the radial shape operator for points corresponding to an edge point $x_{0}$. A smooth value of $U$ in a neighborhood of $x_{0}$ corresponds to one side of $M$. Although $\mathcal{B}_{t}$ is not smooth at $\psi_{t}\left(x_{0}\right)$ if $t<1$, we note that by Proposition 4.4 of [Dam03], provided $1 /(t r)$ is not a generalized eigenvalue for $\left(S_{\mathrm{E} \mathbf{v}}, I_{n-1,1}\right)$, the one side of $\mathcal{B}_{t}$ corresponding to $U$ is smooth and is transverse to $U$ at $x_{0}$ when $t>0$. Thus, the radial shape operator is defined for $\mathcal{B}_{t}$ at points corresponding to edge points when $t>0$. Hence, we may compute the radial shape operator $S_{\mathbf{v}^{\prime} t}$ for this one side as follows.

Proposition 2.3. Suppose that, at an edge point $x_{0} \in \partial M$, we have a smooth value of $U$ (corresponding to one side of $M$ ) and a special basis $\left\{v_{i}\right\}$ for $T_{x_{0}} M$. Let $\left\{v_{i}^{\prime}\right\}$ denote the image of $\left\{v_{i}\right\}$ under $d \psi_{t}\left(x_{0}\right)$. If $1 /(t r)$ is not a generalized eigenvalue of $\left(S_{\mathrm{E} \mathbf{v}}, I_{n-1,1}\right)$, then the radial shape operator $S_{\mathbf{v}^{\prime} t}$ for $\mathcal{B}_{t}$ at $x_{0}^{\prime}=\psi_{t}\left(x_{0}\right)$ is given by

$$
S_{\mathbf{v}^{\prime} t}=\left(I_{n-1,1}-t r \cdot S_{\mathrm{E} \mathbf{v}}\right)^{-1} S_{\mathrm{E} \mathbf{v}} .
$$

We note that, unlike the situation for the radial shape operator, $S_{\mathrm{E} \mathbf{v}}$ does not necessarily commute with $I_{n-1,1}$, so the order of the factors is important.

Proof. For the proof we argue as for Proposition 2.1, using (1.3) and (1.4) instead of (1.1) and (1.2). We compute

$$
\frac{\partial U_{1}}{\partial \mathbf{v}^{\prime}}=A_{U}^{\prime} \cdot U_{1}-S_{\mathrm{E} \mathbf{v}}^{\mathrm{T}}\left(I_{n-1,1}-t r \cdot S_{\mathrm{E} \mathbf{v}}^{\mathrm{T}}\right)^{-1} \mathbf{v}^{\prime}
$$




\section{GeOMETRY OF BOUNDARIES}

where

$$
A_{U}^{\prime}=A_{U}+S_{\mathrm{E} \mathbf{v}}^{\mathrm{T}}\left(I_{n-1,1}-t r \cdot S_{\mathrm{E} \mathbf{v}}^{\mathrm{T}}\right)^{-1} \tilde{A}_{U}
$$

Hence, (2.9) provides an expression for $\partial U_{1} / \partial \mathbf{v}^{\prime}$ in terms of the basis $\mathbf{v}^{\prime}$ and $U_{1}$. Thus, by the definition of $S_{\mathbf{v}^{\prime} t}$ as a matrix representation for $-\operatorname{proj}_{U}\left(\partial U_{1} / \partial v\right)$, where $\operatorname{proj}_{U}$ denotes projection onto $T_{\psi_{t}\left(x_{0}\right)} \mathcal{B}_{t}$ along $U\left(x_{0}\right)$,

implying (2.8).

$$
S_{\mathbf{v}^{\prime} t}^{\mathrm{T}}=S_{\mathrm{E} \mathbf{v}}^{\mathrm{T}}\left(I_{n-1,1}-t r \cdot S_{\mathrm{E} \mathbf{v}}^{\mathrm{T}}\right)^{-1}
$$

Unlike the case of non-edge points, we cannot in general deduce a simple formula for the principal radial curvatures for $S_{\mathbf{v}^{\prime} t}$ in terms of the principal edge curvatures. However, in a special case, we can draw this conclusion.

Example 2.4. Suppose $\partial U_{1} / \partial v_{i}$ is orthogonal to $\mathbf{n}\left(\right.$ i.e. $\left.\in T_{x_{0}} M\right)$ for $i=1, \ldots, n-1$, and $\partial U_{1} / \partial v_{n}$ has a non-zero component for $\mathbf{n}$. Then, the bottom row of $S_{\mathrm{E} \mathbf{v}}$ has the form $(0, \ldots, 0, b)$. Let $\tilde{S}_{\mathrm{E} \mathbf{v}}$ denote the matrix obtained from $S_{\mathrm{E} \mathbf{v}}$ by removing the last row and column. It is the matrix representation for $-\operatorname{proj}_{U}^{\prime \prime}\left(\partial U_{1} / \partial v\right)$, where proj" denotes projection onto $T_{x_{0}} \partial M$ along the subspace $\langle\mathbf{n}, U\rangle$, with respect to the basis $\tilde{\mathbf{v}}=\left\{v_{1}, \ldots, v_{n-1}\right\}$.

Then, for each generalized eigenvalue $\lambda \neq 0$ of $\left(S_{\mathrm{E} \mathbf{v}}, I_{n-1,1}\right)$, the eigenvector $w=\left(w_{1}, \ldots, w_{n}\right)$ must have $w_{n}=0$ and then $\tilde{w}=\left(w_{1}, \ldots, w_{n-1}\right)$ is an eigenvector of $\widetilde{S}_{\mathrm{E} \mathbf{v}}$ with eigenvalue $\lambda$. The converse also holds even for $\lambda=0$. Thus, the $n-1$ generalized eigenvalues $\kappa_{\mathrm{E} i}$ equal the eigenvalues $\tilde{\kappa}_{\mathrm{E} i}$ of $\tilde{S}_{\mathrm{E} \mathbf{v}}$ counting multiplicities. Then, we see that $\left(1-\operatorname{tr} \tilde{\kappa}_{\mathrm{E} i}\right)$ is an eigenvalue of $I_{n-1,1}-t r \cdot S_{\mathrm{E} \mathbf{v}}$ and hence $\left(1-\operatorname{tr} \tilde{\kappa}_{\mathrm{E} i}\right)^{-1} \tilde{\kappa}_{\mathrm{E} i}$ is an eigenvalue of $\left(I_{n-1,1}-t r \cdot S_{\mathrm{E} \mathbf{v}}\right)^{-1} S_{\mathrm{E} \mathbf{v}}$ both with the same eigenvector $(\tilde{w}, 0)$ for the corresponding eigenvector $\tilde{w}$ for $\tilde{S}_{\mathrm{E} \mathbf{v}}$. Also, a direct calculation shows that the standard basis vector $\mathbf{e}_{n}$ is an eigenvector for $\left(I_{n-1,1}-t r \cdot S_{\mathrm{E} \mathbf{v}}\right)^{-1} S_{\mathrm{E} \mathbf{v}}$ with eigenvalue $-1 /(t r)$. These give the principal radial curvatures for $\mathcal{B}_{t}$ at $x_{0}^{\prime}=\psi_{t}\left(x_{0}\right)$ (on the side corresponding to $U$ ) to be

$$
\frac{-1}{t r} \quad \text { and } \quad \kappa_{\mathrm{r} t i}=\frac{\kappa_{\mathrm{E} i}}{\left(1-t r \kappa_{\mathrm{E} i}\right)} \quad i=1, \ldots, n-1,
$$

where $\kappa_{\mathrm{E} i}=\tilde{\kappa}_{\mathrm{E} i}$ for $i=1, \ldots, n-1$.

\section{Differential geometry of the boundary in the Blum case}

We now use the preceding to determine the differential geometry of the boundary in the Blum case. For this we do not even require the full strength of the Blum conditions for the Blum medial axis. Recall for a smooth value of $U$ defined on a local component of $M$ for $x_{0}$, the compatibility 1-form $\eta_{U}(v)=v \cdot U_{1}+d r(v)$; and the compatibility condition at $x_{0}$ (for the given choice of $U$ ) asserts $\eta_{U} \equiv 0$ at $x_{0}$. We begin with a simple consequence of the compatibility condition holding at a point $x_{0}$. Then, as in Lemma 6.1 of [Dam03] we have the following lemma.

Lemma 3.1. Suppose $(M, U)$ is a skeletal structure and that $x_{0} \in M$ is a non-edge point. Let $U$ be a smooth value (on a non-edge local manifold component $M_{\beta}$ of $x_{0}$ ) for which $1 / r$ is not an eigenvalue of $S_{\mathrm{rad}}$ at $x_{0}$. Then, the corresponding compatibility 1-form $\eta_{U}$ vanishes at $x_{0}$ if and only if $U\left(x_{0}\right)$ is orthogonal to the associated boundary at $\psi_{1}\left(x_{0}\right)$.

Proof. By the proof of Proposition 4.1 of part I, as $1 / r$ is not an eigenvalue of $S_{\text {rad }}$, it follows that $\psi_{1}$ is a local diffeomorphism. We choose a neighborhood $W$ of the local manifold component $M_{\beta}$ so that $\psi_{1}$ is a diffeomorphism on $W$. For $v \in T_{x_{0}} M_{\beta}$, we compute the dot product $\left(\partial \psi_{1} / \partial v\right) \cdot U_{1}$. Recall that

$$
\frac{\partial \psi_{t}}{\partial v}=v+t\left(\frac{\partial r}{\partial v} \cdot U_{1}+r \cdot \frac{\partial U_{1}}{\partial v}\right)
$$




\section{J. DAMON}

Then, we have

$$
\frac{\partial \psi_{1}}{\partial v} \cdot U_{1}=v \cdot U_{1}+d r(v)\left(U_{1} \cdot U_{1}\right)+r\left(\frac{\partial U_{1}}{\partial v} \cdot U_{1}\right) .
$$

Since differentiating $U_{1} \cdot U_{1}=1$ implies $\left(\partial U_{1} / \partial v\right) \cdot U_{1}=0$, we obtain

$$
\frac{\partial \psi_{1}}{\partial v} \cdot U_{1}=v \cdot U_{1}+d r(v)=\eta_{U}(v) .
$$

Thus, $U_{1}$ being orthogonal to $\partial \psi_{1} / \partial v$ for all $v$ is equivalent to $\eta_{U} \equiv 0$ at $x_{0}$.

Thus, $U_{1}\left(x_{0}^{\prime}\right)$, which is also the translate of $U_{1}$ along the line spanned by $U\left(x_{0}\right)$, is orthogonal to $T_{x_{0}^{\prime}} \mathcal{B}$, where $x_{0}^{\prime}=\psi_{1}\left(x_{0}\right)$.

Thus, if, for a smooth value of $U$ on an open set $W$ of smooth points of $M$, the compatibility condition holds for $\eta_{U}$ on all of $W$, then by Lemma 3.1, $U$ is orthogonal to the associated boundary on $\tilde{W}=\psi_{1}(W)$. Thus, we see that the radial shape operator at a point $x^{\prime} \in \tilde{W}$ of the boundary is really the differential geometric shape operator for $\mathcal{B}$ at $x^{\prime}$. Hence, we can combine our calculation of the radial shape operator at $x_{0}^{\prime}=\psi_{1}\left(x_{0}\right)$ given by Proposition 2.1 with Lemma 3.1 to compute the differential geometric shape operator, principal curvatures, and principal directions of $\mathcal{B}$ in terms of the radial shape operator, principal radial curvatures, and principal radial directions.

\section{Differential geometry at associated smooth points}

We consider a smooth point $x_{0} \in M$ (however, we could equally well consider a local component for a non-edge singular point of $M$ ).

Theorem 3.2. Suppose $(M, U)$ is a skeletal structure such that, for a choice of smooth value of $U$, the associated compatibility 1-form $\eta_{U}$ vanishes identically on a neighborhood of a smooth point $x_{0}$ of $M$, and $1 / r$ is not an eigenvalue of $S_{\mathrm{rad}}$ at $x_{0}$. Let $x_{0}^{\prime}=\psi_{1}\left(x_{0}\right)$, and $\mathbf{v}^{\prime}$ be the image of $\mathbf{v}$ for a basis $\left\{v_{1}, \ldots, v_{n}\right\}$.

1) The differential geometric shape operator $S_{\mathcal{B}}$ of $\mathcal{B}$ at $x_{0}^{\prime}$ has a matrix representation with respect to $\mathbf{v}^{\prime}$ given by

$$
S_{\mathcal{B} \mathbf{v}^{\prime}}=\left(I-r \cdot S_{\mathbf{v}}\right)^{-1} S_{\mathbf{v}} .
$$

2) Hence, there is a bijection between the principal curvatures $\kappa_{i}$ of $\mathcal{B}$ at $x_{0}^{\prime}$ and the principal radial curvatures $\kappa_{\mathrm{r} i}$ of $M$ at $x_{0}$ (counted with multiplicities) given by

$$
\kappa_{i}=\frac{\kappa_{\mathrm{r} i}}{\left(1-r \kappa_{\mathrm{r} i}\right)} \quad \text { or equivalently } \quad \kappa_{\mathrm{r} i}=\frac{\kappa_{i}}{\left(1+r \kappa_{i}\right)} .
$$

3) Furthermore, the principal radial directions corresponding to $\kappa_{\mathrm{r} i}$ are mapped by $d \psi_{1}$ to the principal directions corresponding to $\kappa_{i}$.

Proof. By Lemma 3.1, the radial vector field $U$ is orthogonal to the boundary at points in a neighborhood of $x_{0}^{\prime}=\psi_{1}\left(x_{0}\right)$. Thus, the radial shape operator at a point $x_{0}^{\prime}$ is really the differential geometric shape operator for $\mathcal{B}$ at $x_{0}^{\prime}$. Hence, we can apply our calculation of the radial shape operator at $x_{0}^{\prime}$ given by Proposition 2.1. We deduce that the radial shape operator, principal radial curvatures, and principal radial directions for $S_{\operatorname{rad} t}$ at $x_{0}^{\prime}$ for $t=1$ are really the differential geometric shape operator, principal curvatures, and principal directions for $\mathcal{B}$ at $x_{0}^{\prime}$.

We obtain the following corollary for Blum medial axes.

Corollary 3.3. Suppose $\Omega$ is a region in $\mathbb{R}^{n+1}$ with smooth boundary $\mathcal{B}$ and Blum medial axis and radial vector field $(M, U)$. Let $x_{1} \in \mathcal{B}$ be a point for which the projection onto the medial axis along normals is a local diffeomorphism (with $x_{1}$ mapping to $x_{0} \in M$ ). Then, the differential geometric 


\section{GEOMETRY OF BOUNDARIES}

shape operator for $\mathcal{B}$ at $x_{1}$, the principal normal curvatures and principal directions are given by the corresponding radial shape operator for $(M, U)$ at $x_{0}$, together with its principal curvatures and principal directions, as in Theorem 3.2.

Proof. The Blum condition implies that $U$ is orthogonal to $\mathcal{B}$, which is the boundary of the medial axis $M$ with radial vector field $U$. Let $x_{1} \in \mathcal{B}$ be a point for which the projection onto the medial axis along normals is a local diffeomorphism (with $x_{1}$ mapping to $x_{0} \in M$ ). Then, the inverse of the projection which is given by $\psi_{1}$ is also a local diffeomorphism. Thus, $1 / r$ is not an eigenvalue of $S_{\text {rad }}$ for the corresponding smooth value of $U$. Hence, we can apply Theorem 3.2 to obtain the results.

Remark 3.4 (Geometry at points corresponding to singular points). In the situation of Corollary 3.3,

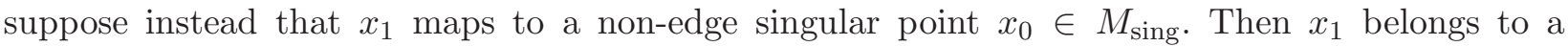
local complementary component $C_{i}$ of $x_{0}$. Let $M_{\alpha}$ be a local component for $x_{0}$ which belongs to $\partial C_{i}$. Then $M_{\alpha}$ together with the smooth value of $U$ which points into $C_{i}$ extend smoothly on a neighborhood of $x_{0}$. The corresponding differential geometric shape operator for $\mathcal{B}$ at $x_{1}$, the principal curvatures, and principal directions are again given by the corresponding radial shape operator for the corresponding smooth choice of $U$ on $M_{\alpha}$, together with its principal curvatures and principal directions. For the Blum medial axis, these must match up for the various choices of local components $M_{\alpha}$ for $x_{0}$ belonging to $\partial C_{i}$.

As an immediate consequence, we deduce the Riemannian curvature for the boundary using Gauss's formula (see e.g. [Spi75, vol. III]).

COROLlary 3.5. Let $\Omega \subset \mathbb{R}^{n+1}$ be a region with smooth boundary and Blum medial axis and radial vector field $(M, U)$. Let $x_{1} \in \mathcal{B}$ be a point at which projection onto the medial axis along normals is a local diffeomorphism (with $x_{1}$ mapping to $x_{0} \in M$ ). The Riemannian curvature of $\mathcal{B}$ is given with respect to basis $\mathbf{v}^{\prime}$, corresponding to the basis $\mathbf{v}$ at $x_{0}$, by

$$
R(v, w)(u)=\langle\bar{w}, u\rangle \bar{v}-\langle\bar{v}, u\rangle \bar{w},
$$

where $\bar{v}=\left(I-r S_{\mathbf{v}}\right)^{-1} S_{\mathbf{v}} v$, etc.

Hence, all intrinsic geometry of the boundary $\mathcal{B}$ is determined by the radial shape operator. In the special case of surfaces in $\mathbb{R}^{3}$, this reduces to multiplication by the determinant of $S_{\mathcal{B}}$, which is the Gauss curvature. In fact, in [Dam04] we further show that, as a result of the radial curvature condition, the signs of the principal and the Gauss curvatures are determined by the signs of $\kappa_{\mathrm{r} i}$ and $\operatorname{det}\left(S_{\mathbf{v}}\right)$.

By contrast the 'relative geometry' of $\mathcal{B}$ is more subtle and depends on more than just $S_{\mathbf{v}}$. For example, if we wish to compare principal curvatures $\kappa_{i}$ at different points or to determine rates of change of $\kappa_{i}$ along curves we cannot do it solely using $\kappa_{\mathrm{r} i}$. The relation is most easily expressed using the signed radii of curvatures $r_{i}=1 / \kappa_{i}$ and the signed radii of radial curvatures $r_{\mathrm{r} i}=1 / \kappa_{\mathrm{r}} i$. We may reexpress the relation (3.4) in the following simple radii of curvatures equation:

$$
r_{\mathrm{r} i}=r+r_{i} .
$$

This reveals at the level of eigenvalues the basic relation between $S_{\mathcal{B} \mathbf{v}^{\prime}}$ and $S_{\mathbf{v}}$ given by (3.3) which can be rewritten in the case $S_{\mathbf{v}}$ is invertible as

$$
S_{\mathbf{v}}^{-1}=r \cdot I+S_{\mathcal{B} \mathbf{v}^{\prime}}^{-1}
$$

Then (3.6) allows us to relate the properties of $r_{i}$ at different points and the rate of change of $r_{i}$ along various curves in terms of the corresponding changes for $r_{\mathrm{r} i}$, but such comparison must involve $r$ or its derivatives. We explicitly determine in [Dam04] both the intrinsic and relative 


\section{J. DAMON}

geometry for surfaces in $\mathbb{R}^{3}$ using the radial (and edge) shape operators, the principal radial (and edge) curvatures, and for the relative geometry the 'relative critical set' of $r$.

\section{Differential geometry at crest points (associated to edge points)}

In the partial Blum case, Theorem 3.2 allows us to compute the differential geometric shape operator at smooth points of $M$. Hence, for a crest point of $M$ which corresponds to an edge point $x_{0}$ under the radial map, we could determine the geometry at a point on the boundary by taking the limit as $x \rightarrow x_{0}$ of $S_{\mathcal{B} \mathbf{v}^{\prime}}=\left(I-r \cdot S_{\mathbf{v}}\right)^{-1} S_{\mathbf{v}}$. The problem with this is the unstable nature of $S_{\mathbf{v}}$ as we approach the edge of $M . S_{\text {rad }}$ is computed by projection onto $T_{x} M$ along $U$. As we approach the edge, the angle between $U$ and $T_{x} M$ approaches 0 , so the projection increasingly becomes numerically unstable. It would be preferable to compute the shape operator at a crest point using the edge shape operator because it involves projection along $U$ but onto the subspace $T_{x_{0}} \partial M \oplus\langle\mathbf{n}\rangle$. Generically $U$ will be bounded away from this subspace providing a lower bound for the stability of the projection. We consider a smooth value $U$ corresponding to one side of $M$ near an edge point $x_{0}$. Then, we can obtain the analogue of Corollary 3.3 for crest points.

Corollary 3.6. Suppose $\Omega$ is a region in $\mathbb{R}^{n+1}$ with smooth boundary $\mathcal{B}$ and Blum medial axis and radial vector field $(M, U)$. Let $x_{1} \in \mathcal{B}$ be a crest point corresponding to an edge point $x_{0} \in \partial M$. We let $\mathbf{v}$ be a special basis for $T_{x_{0}} M$ with $\mathbf{v}^{\prime}$ the corresponding basis for $T_{x_{1}} \mathcal{B}$. Then, the differential geometric shape operator for $\mathcal{B}$ at $x_{1}$ has a matrix representation with respect to $\mathbf{v}^{\prime}$ given by

$$
S_{\mathcal{B} \mathbf{v}^{\prime}}=\left(I_{n-1,1}-r \cdot S_{\mathrm{E} \mathbf{v}}\right)^{-1} S_{\mathrm{E} \mathbf{v}} .
$$

Hence, the principal curvatures $\kappa_{i}$ and principal directions of $\mathcal{B}$ at $x_{1}$ are the eigenvalues and eigendirections (after identification) of the right-hand side of (3.3).

Proof. By the Blum condition, the $U$ will be orthogonal to $\mathcal{B}$. Thus, the radial shape operator at a point will, in fact, be the differential geometric shape operator. Then, we can apply Proposition 2.3 to obtain the stated form for $S_{\mathcal{B} \mathbf{v}^{\prime}}$, and then the corresponding eigenvalues and eigendirections.

Example 3.7. We return to Example 2.4, except that now we suppose $M$ is the Blum medial axis of $\mathcal{B}$. We consider a crest point $x_{1}$ which corresponds to a point $x_{0} \in \partial M$ for which $\partial U_{1} / \partial v_{i}$ is orthogonal to $\mathbf{n}$ for $i=1, \ldots, n-1$, and $\partial U_{1} / \partial v_{n}$ has a non-zero component for $\mathbf{n}$. Then, by Example 2.4, we conclude that, at the corresponding crest point $x_{1}$, the principal curvatures of $\mathcal{B}$ are

$$
-\frac{1}{r} \quad \text { and } \quad \kappa_{i}=\frac{\kappa_{\mathrm{E} i}}{\left(1-r \kappa_{\mathrm{E} i}\right)} \quad i=1, \ldots, n-1,
$$

where the $\kappa_{\mathrm{E} i}$ are the principal edge curvatures.

Using Corollary 3.6 and the edge shape operator to compute the principal curvatures at a crest point $x_{1}$ is difficult because the computation requires the use of edge coordinates which are typically not known when given a Blum medial axis. There is still a way to compute the principal curvatures as limits but now coming from a stable computation.

We let $\left\{v_{i}\right\}$ denote a local frame for $M$ in a neighborhood $W$ of $x_{0}$, an edge point corresponding to $x_{1}$. For $i=1, \ldots, n-1$, we choose the $v_{i}$ to be tangent to $T \partial M$ and smooth relative to local coordinates for $M$ a manifold with boundary (they are then also smooth with respect to edge coordinates). We also let $v_{n}=U_{1 \tan }$, the tangential component of $U_{1}$. It is smooth with respect to edge coordinates. By possibly shrinking $W$, we may assume that the $v_{i}$ together with $\mathbf{n}$ will form a frame for $\mathbb{R}^{n+1}$ at each point of $W$. We can define an operator $S_{\mathrm{E}}^{\prime}$ at each point of $W$ and extend $S_{\mathrm{E}}$ as follows:

$$
S_{\mathrm{E}}^{\prime}(v)=-\operatorname{proj}_{U}^{\prime \prime}\left(\frac{\partial U_{1}}{\partial v}\right)
$$




\section{GEOMETRY OF BOUNDARIES}

where now $\operatorname{proj}_{U}^{\prime \prime}$ denotes projection along $U$ but onto $L$, the subspace spanned by $\left\{v_{1}, \ldots, v_{n-1}, \mathbf{n}\right\}$. We note that, at a non-edge point $x$ near $x_{0}$, the difference between $S_{\text {rad }}$ and $S_{\mathrm{E}}^{\prime}$ is just the subspace to which we project. For $S_{\mathrm{rad}}$ it is $T_{x} M$, while for $S_{\mathrm{E}}^{\prime}$ it is $L$. However, as $x \rightarrow x_{0}, L \rightarrow T_{x_{0}} M$. We let $S_{\mathrm{E} \mathbf{v}}^{\prime}$ denote the matrix representation of $S_{\mathrm{E}}^{\prime}$ with respect to the basis $\mathbf{v}$ in the source and $\left\{v_{1}, \ldots, v_{n-1}, \mathbf{n}\right\}$ in the target. If $S_{\mathbf{v}^{\prime} t}$ denotes the matrix representation of the radial shape operator on $\mathcal{B}_{t}$ at $x_{0}^{\prime}=\psi_{t}\left(x_{0}\right)$ with respect to the corresponding basis $\mathbf{v}^{\prime}$, then it can be computed as a limit.

LEMMA 3.8. In the preceding situation,

$$
S_{\mathbf{v}^{\prime} t}=\lim _{x \rightarrow x_{0}}\left(I_{n-1,1}-t r \cdot S_{\mathrm{E} \mathbf{v}}^{\prime}\right)^{-1} S_{\mathrm{E} \mathbf{v}}^{\prime} .
$$

Proof. The same argument used to compute (2.8) in Proposition 2.3 shows that, at $x^{\prime}=\psi_{t}(x)$ for $x$ in a small neighborhood of $x_{0}$,

$$
S_{\mathbf{v}^{\prime} t}=\left(I_{n-1,1}-t r \cdot S_{\mathrm{E} \mathbf{v}}^{\prime}\right)^{-1} S_{\mathrm{E} \mathbf{v}}^{\prime} .
$$

In (3.12), we take the limit as $x \rightarrow x_{0}$. By continuity $S_{\mathrm{E} \mathbf{v}}^{\prime} \rightarrow S_{\mathrm{E} \mathbf{v}}$, so we obtain

$$
\lim _{x \rightarrow x_{0}}\left(I_{n-1,1}-t r \cdot S_{\mathrm{E} \mathbf{v}}^{\prime}\right)^{-1} S_{\mathrm{E} \mathbf{v}}^{\prime}=\left(I_{n-1,1}-t r \cdot S_{\mathrm{E} \mathbf{v}}\right)^{-1} S_{\mathrm{E} \mathbf{v}} .
$$

By Proposition 2.3, the right-hand side of (3.13) is $S_{\mathbf{v}^{\prime} t}$, yielding (3.11).

We deduce the following as a consequence.

COROLlary 3.9. The principal curvatures of $\mathcal{B}$ at a crest point $x_{1}$ corresponding to $x_{0} \in \partial M$ (which are the eigenvalues of $S_{\mathcal{B} \mathbf{v}^{\prime}}$ ) are the limits as $x \rightarrow x_{0}$ of the eigenvalues of

$$
\left(I_{n-1,1}-r \cdot S_{\mathrm{E} \mathbf{v}}^{\prime}\right)^{-1} S_{\mathrm{E} \mathbf{v}}^{\prime}
$$

Moreover, if the principal curvatures at $x_{0}$ are distinct, then the principal directions are the limits of the eigendirections of (3.14) as $x \rightarrow x_{0}$.

Hence, the principal curvatures at $x_{1}$ (and principal directions in the generic case) can be approximated by the eigenvalues of $\left(I_{n-1,1}-r \cdot S_{\mathrm{E} \mathbf{v}}^{\prime}\right)^{-1} S_{\mathrm{E} \mathbf{v}}^{\prime}$ (respectively, the eigendirections) for $x$ sufficiently close to $x_{0}$.

\section{Relation between the radial and differential geometry of the skeletal set in the Blum case}

In the preceding section we established in the (partial) Blum case the fundamental relation between the radial geometry on the skeletal set and the differential geometry of the boundary. By contrast, in the Introduction we referred to work in the Blum case relating the differential geometry of the boundary and that of the skeletal set using derivative information of the radial function $r$. We derive an explicit relation in the (partial) Blum case between the differential geometric shape operator of the skeletal set and the radial shape operator.

Let $(M, U)$ be a skeletal structure which satisfies the compatibility condition on an open set $W \subset M_{\text {reg. }}$. Then, as a submanifold of $\mathbb{R}^{n+1}, M_{\text {reg }}$ has a Riemannian structure. We let $S_{\text {med }}$ denote the differential geometric shape operator of $M$ ('med' is to remind us that in the Blum case $M$ is the Blum medial axis). To obtain a relation, we will introduce two other operators on $W$. To do so we decompose $U_{1}$ into tangential and normal components $U_{1}=U_{1 \tan }+\rho \mathbf{n}$, where $\mathbf{n}$ is a unit normal vector field pointing to the same side as the smooth value of $U$. We let $\nabla f$ denote the Riemannian gradient (so that $\langle\nabla f, v\rangle=d f(v)$ ). Then, by the compatibility condition, $d r(v)=$ $-\left\langle U_{1}, v\right\rangle=\left\langle-U_{1 \tan }, v\right\rangle$. Thus, $\nabla r=-U_{1 \text { tan }}$. Hence, $\rho=\left(1-\|\nabla r\|^{2}\right)^{1 / 2}$, which we can think of as the 'normal component function for $U_{1}$ '. 


\section{J. DAMON}

The Riemannian Hessian is defined by $H(f)(v, w) \stackrel{\text { def }}{=}\left\langle\nabla_{v}(\nabla f), w\right\rangle$. Here $\nabla_{v}$ denotes the covariant derivative of the vector field $\nabla f$ (we recall that, for a vector field $X, \nabla_{v} X\left(x_{0}\right)=\operatorname{proj}_{\mathbf{n}}(\partial X / \partial v$ ) where $\operatorname{proj}_{\mathbf{n}}$ denotes orthogonal projection onto $\left.T_{x_{0}} N\right)$. By properties of the covariant derivative, $H(f)$ is symmetric in $v$ and $w$. We define the Hessian operator $H_{f}: T_{x_{0}} N \rightarrow T_{x_{0}} N$ by $H_{f}(v)=\nabla_{v}(\nabla f)\left(x_{0}\right)$. As $\left\langle H_{f}(v), w\right\rangle=H(f)(v, w)$, it follows that $H_{f}$ is self-adjoint. We are interested in the radial Hessian operator $H_{\mathrm{r}}$. In the (partial) Blum case, $H_{\mathrm{r}}(v)=-\nabla_{v} U_{1 \mathrm{tan}}$.

One further operator must be defined. We let

$$
Z(v)=\rho^{-1}\left(\frac{\partial U_{1}}{\partial v} \cdot \mathbf{n}\right) U_{1 \tan }
$$

Unlike the case of $S_{\text {med }}$ and $H_{\mathrm{r}}, Z$ need not be self-adjoint. Also, $Z$ does not have an obvious geometric meaning. Nonetheless, there is the following relation between the radial and the differential geometric shape operators.

Proposition 4.1. Let $(M, U)$ be a skeletal structure which satisfies the compatibility condition on an open set $W \subset M_{\text {reg. }}$. Let $U$ be a smooth value on $W$. Then, on $W$ there is the following relation:

$$
S_{\text {rad }}=\rho \cdot S_{\text {med }}+H_{\mathrm{r}}+Z \text {. }
$$

Before deriving this relation, we note two consequences. We first see that, although $S_{\text {med }}$ and $H_{\mathrm{r}}$ are self-adjoint, $Z$ measures the failure of $S_{\mathrm{rad}}$ to be self-adjoint. Second, given that we have derived in Theorem 3.2 the simple relations between the differential geometric shape operator of the associated boundary and radial shape operators $S_{\mathcal{B}}$ and $S_{\text {rad }}$, there follows from (3.4) combined with (4.2) the relation between $S_{\mathcal{B}}$ and $S_{\text {med }}$. In this relation, it is necessary to include both $H_{\mathrm{r}}$ and $Z$ as well as $\rho$. Hence, we see that using $S_{\text {rad }}$ instead of $S_{\text {med }}$ leads to a more natural relation with the differential geometric shape operator of the boundary.

Proof. We are comparing

$$
S_{\mathrm{rad}}(v)=-\operatorname{proj}_{U}\left(\frac{\partial U_{1}}{\partial v}\right) \quad \text { and } \quad S_{\mathrm{med}}(v)=-\operatorname{proj}_{\mathbf{n}}\left(\frac{\partial \mathbf{n}}{\partial v}\right) .
$$

In addition, we have

$$
H_{\mathrm{r}}(v)=-\nabla_{v} U_{1 \tan }=-\operatorname{proj}_{\mathbf{n}}\left(\frac{\partial U_{1 \tan }}{\partial v}\right) .
$$

Thus, we first compare $\operatorname{proj}_{U}$ and $\operatorname{proj}_{\mathbf{n}}$.

Let $\mathbf{w}=a_{\mathbf{w}} \mathbf{n}+\tilde{\mathbf{w}}$ be the decomposition into tangential and normal components. Then, $\operatorname{proj}_{\mathbf{n}}(\mathbf{w})$ $=\tilde{\mathbf{w}}$. By the decomposition of $U_{1}, \mathbf{n}=\rho^{-1}\left(U_{1}-U_{1 \tan }\right)$. Thus, we can decompose $\mathbf{w}$ by

$$
\begin{aligned}
\mathbf{w} & =a_{\mathbf{w}} \rho^{-1}\left(U_{1}-U_{1 \tan }\right)+\tilde{\mathbf{w}} \\
& =a_{\mathbf{w}} \rho^{-1} \cdot U_{1}+\left(\tilde{\mathbf{w}}-a_{\mathbf{w}} \rho^{-1} \cdot U_{1 \tan }\right) .
\end{aligned}
$$

Thus, from (4.3), $\operatorname{proj}_{U}(\mathbf{w})=\tilde{\mathbf{w}}-a_{\mathbf{w}} \rho^{-1} \cdot U_{1 \tan }$. Hence,

$$
\operatorname{proj}_{\mathbf{n}}(\mathbf{w})=\operatorname{proj}_{U}(\mathbf{w})+a_{\mathbf{w}} \rho^{-1} \cdot U_{1 \tan } .
$$

Then,

$$
S_{\mathrm{rad}}(v)=-\operatorname{proj}_{U}\left(\frac{\partial U_{1}}{\partial v}\right)=-\operatorname{proj}_{\mathbf{n}}\left(\frac{\partial U_{1}}{\partial v}\right)+a \rho^{-1} \cdot U_{1 \tan },
$$

where $a$ denotes the normal coefficient of $\partial U_{1} / \partial v$.

Then, from the decomposition for $U_{1}$, we compute

$$
\frac{\partial U_{1}}{\partial v}=\frac{\partial U_{1} \tan }{\partial v}+d \rho(v) \cdot \mathbf{n}+\rho \cdot \frac{\partial \mathbf{n}}{\partial v} .
$$


Thus, from (4.6)

$$
\begin{aligned}
-\operatorname{proj}_{\mathbf{n}}\left(\frac{\partial U_{1}}{\partial v}\right) & =-\operatorname{proj}_{\mathbf{n}}\left(\frac{\partial U_{1 \tan }}{\partial v}\right)+0-\rho \cdot \operatorname{proj}_{\mathbf{n}}\left(\frac{\partial \mathbf{n}}{\partial v}\right) \\
& =-\nabla_{v} U_{1 \tan }+\rho \cdot S_{\text {med }}(v) \\
& =H_{\mathrm{r}}(v)+\rho \cdot S_{\text {med }}(v) .
\end{aligned}
$$

We substitute (4.7) into (4.5) and observe that as $a=\left(\partial U_{1} / \partial v\right) \cdot \mathbf{n}$, the last term in (4.5) is $Z(v)$. This yields $(4.2)$.

\section{Effects of a diffeomorphism of the skeleton on the smoothness of boundaries}

We are now in a position to consider the effect of a diffeomorphism of a skeletal structure on the associated boundary as in Figure 2.
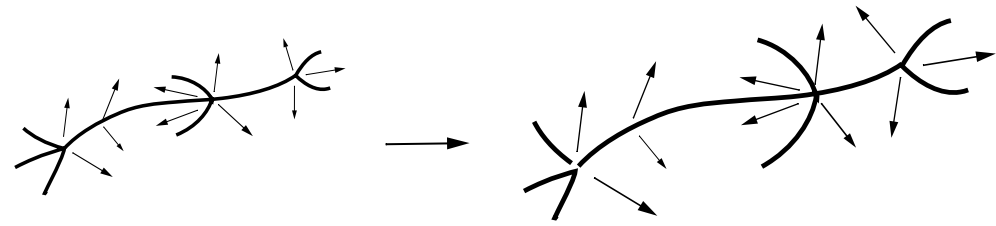

FiguRE 2. Diffeomorphism of a skeletal structure.

Definition 5.1. A diffeomorphism of skeletal structures $\varphi:(M, U) \rightarrow\left(M^{\prime}, V\right)$ consists of a diffeomorphism $\varphi$ from an open neighborhood $W$ of $M$ to an open subset of $\mathbb{R}^{n+1}$ such that $M^{\prime}=\varphi(M)$ and $V=d \varphi(U)$.

Even though such a $\varphi$ is defined geometrically, it is only defined on a small neighborhood of $M$; hence it does not guarantee any properties of the associated boundary of the image skeletal structure.

We ask how the radial shape operators, edge shape operators, and compatibility 1-forms compare so we can determine the effects of $\varphi$ on the curvature, edge and compatibility conditions and also on the geometry of the associated boundary.

We let $V=r_{1} \cdot V_{1}$, and $V_{1}(\varphi(x))=\sigma(x) \cdot d \varphi_{x}\left(U_{1}(x)\right)$. We call $\sigma$ the radial scaling factor. As with $U$ and $r, \sigma$ is multivalued with a value at $x$ for each value of $U$ at $x$. Then,

$$
V=r_{1} \cdot V_{1}=r_{1}(\varphi(x)) \sigma(x) d \varphi_{x}\left(U_{1}(x)\right)=d \varphi_{x}\left(r_{1} \sigma(x) U_{1}(x)\right) .
$$

Hence, we conclude that $r_{1}(\varphi(x)) \sigma(x) \cdot U_{1}(x)=r(x) \cdot U_{1}(x)$ or in abbreviated form $r=\sigma \cdot r_{1} \circ \varphi$.

\section{Compatibility conditions}

We really consider compatibility conditions in two different contexts: for points of $M_{\text {sing }}$ as one of the three conditions to ensure smoothness of the associated boundary, and on open subsets of $M$ to ensure the partial Blum condition for the associated boundary. We wish to identify a natural condition which guarantees that either of these conditions is preserved by $\varphi$.

We begin by determining the effect of $\varphi$ on the compatibility 1-form. We use this to identify a special case when the compatibility 1-forms behave well under pull-back by $\varphi$ (or equivalently under push-forward by $\varphi$ ). Let $\eta_{V}$ be the compatibility 1-form for $\left(M^{\prime}, V\right)$, and let $x_{0}^{\prime}=\varphi\left(x_{0}\right)$.

Definition 5.2. A diffeomorphism of skeletal structures $\varphi:(M, U) \rightarrow\left(M^{\prime}, V\right)$ will be said to be radially rigid at $\left(x_{0}, U_{0}\right)$ if

$$
d \varphi\left(U_{0}\right) \cdot d \varphi(v)=U_{0} \cdot v
$$

for all $v \in \mathbb{R}^{n+1}$; and in addition $d \sigma\left(x_{0}\right) \equiv 0$. 


\section{J. DAMON}

In particular, this implies that $\left\|d \varphi\left(U_{0}\right)\right\|=\left\|U_{0}\right\|$ and that the dot product of $U_{0}$ with vectors in $T_{x_{0}} M_{\beta}$ is preserved for each local component $M_{\beta}$ on which the value $U_{0}$ extends smoothly. In terms of the preceding notation, it follows that $\sigma\left(x_{0}\right)=1$. If $\varphi$ is radially rigid on an open set, of smooth points, then $\sigma \equiv 1$ on the open set, so $d \sigma \equiv 0$, and the second condition is superfluous.

We begin with a simple consequence of $\varphi$ being radially rigid.

Lemma 5.3. Let $\varphi:(M, U) \rightarrow\left(M^{\prime}, V\right)$ be a diffeomorphism of skeletal structures. Suppose $U$ is a smooth value on a local component for $x_{0}$ with $V$ the corresponding image value at $x_{0}^{\prime}=\varphi\left(x_{0}\right)$. If $\varphi$ is radially rigid at $x_{0}$ then $\varphi^{*} \eta_{V}=\eta_{U}$ at $x_{0}$. Hence, if $\varphi$ is radially rigid at $x_{0}$ and the compatibility condition holds for $\eta_{U}$ at $x_{0}$, then it holds for $\eta_{V}$ at $x_{0}^{\prime}$.

In particular, if both $\varphi$ is radially rigid and $(M, U)$ satisfies the compatibility condition on an open set $W$, then so does $\left(M^{\prime}, V\right)$ satisfy the compatibility condition on $\varphi(W)$.

Proof. To be specific, suppose the smooth value $U$ is defined on the local component $M_{\beta}$ of $x_{0}$. We consider the corresponding component for $M_{\beta}^{\prime}=\varphi\left(M_{\beta}\right)$. We let $v^{\prime}=d \varphi(v)$ for $v \in T_{x_{0}} M_{\beta}$. Then,

$$
\begin{aligned}
\eta_{V}\left(v^{\prime}\right) & =V_{1} \cdot v^{\prime}+d r_{1}\left(v^{\prime}\right) \\
& =\sigma d \varphi\left(U_{1}\right) \cdot d \varphi(v)+d r_{1}(d \varphi(v))
\end{aligned}
$$

or as $r_{1} \circ \varphi=r / \sigma$

$$
\eta_{V}\left(v^{\prime}\right)=\sigma d \varphi\left(U_{1}\right) \cdot d \varphi(v)+\left(\frac{1}{\sigma} d r(v)-\frac{r}{\sigma^{2}} d \sigma(v)\right) .
$$

However, by radial rigidity $\sigma\left(x_{0}\right)=1$ (so $\left.r_{1}\left(x_{0}^{\prime}\right)=r \circ \varphi\left(x_{0}\right)\right)$ and $d \sigma\left(x_{0}\right) \equiv 0$. Hence, (5.1) simplifies to

$$
\begin{aligned}
\eta_{V}\left(v^{\prime}\right) & =d \varphi\left(U_{1}\right) \cdot d \varphi(v)+d r(v) \\
& =\left(d \varphi\left(U_{1}\right) \cdot d \varphi(v)-U_{1} \cdot v\right)+\eta_{U}(v) .
\end{aligned}
$$

Then, first $\varphi$ is radially rigid at $x_{0}$ if and only if the first term on the right-hand side of (5.2) is zero for all $v \in T_{x_{0}} M_{\beta}$. This term in (5.2) is zero for all $v \in T_{x_{0}} M_{\beta}$ if and only if $\varphi^{*} \eta_{V}\left(x_{0}\right)=\eta_{U}\left(x_{0}\right)$ on $M_{\beta}$.

If $\eta_{U}\left(x_{0}\right)=0$ for a local component $M_{\beta}$, then, as $d\left(\varphi \mid M_{\beta}\right)\left(x_{0}\right)$ is an isomorphism, $\eta_{V}=0$ on $T_{x_{0}^{\prime}} \varphi\left(M_{\beta}\right)$.

Hence, a diffeomorphism $\varphi:(M, U) \rightarrow\left(M^{\prime}, V\right)$ of skeletal structures which is radially rigid at all points preserves the compatibility condition in that $\left(M^{\prime}, V\right)$ will satisfy the compatibility condition on all open sets $\varphi(W)$ for which $(M, U)$ satisfies it on $W$.

We should remark that being radially rigid at all points is a fairly restrictive condition in that $V$ will have the same length as $U$ at all corresponding points and have the same relative position to the tangent spaces. For example, if on some open $W \subset M_{\text {reg }}, U$ is a normal vector field with $\|U\|$ constant, then the associated boundary to $M$ corresponding to $W$ will consist of parallel manifolds of $M$. If $\varphi$ is radially rigid on $W$, then $V$ will also be a normal vector field of constant length $\|V\|(=\|U\|)$ so the associated boundary for $\left(M^{\prime}, V\right)$ will also consist of parallel manifolds of $M^{\prime}$ for $\varphi(W)$. However, the diffeomorphism $\varphi \mid W$ is otherwise arbitrary. 


\section{GeOMETRY OF BOUNDARIES}

\section{Radial and edge distortion operators}

We secondly examine how a diffeomorphism of skeletal structures $\varphi$ modifies the radial and edge shape operators. We do so by introducing operators which measure the radial and edge distortion due to $\varphi$. Let $x_{0}^{\prime}=\varphi\left(x_{0}\right)$ and $M_{\beta}^{\prime}=\varphi\left(M_{\beta}\right)$. For a linear operator $A: T_{x_{0}} M_{\beta} \rightarrow T_{x_{0}^{\prime}} M_{\beta}^{\prime}$, we use $d \varphi$ to define an associated operator $\left(d \varphi_{x_{0}}\right)^{-1} \circ A$ from $T_{x_{0}} M_{\beta}$ to itself.

We define the radial distortion operator $Q_{\varphi}$ for non-edge points $x_{0}$ of $M$ and smooth value $U$ on $M_{\beta}$ by

$$
Q_{\varphi}(v)=-d \varphi^{-1}\left(\operatorname{proj}_{V}\left(d^{2} \varphi_{x_{0}}\left(v, U_{1}\right)\right)\right)
$$

where $\operatorname{proj}_{V}$ denotes projection along $V$ onto $T_{x_{0}^{\prime}} M_{\beta}^{\prime}$. To obtain a matrix representation for a basis $\left\{v_{1}, \ldots, v_{n}\right\}$ with $v_{i}^{\prime}=d \varphi\left(v_{i}\right)$ for all $i$, let

$$
d^{2} \varphi_{x_{0}}\left(v_{i}, U_{1}\right)=a_{\varphi i} \cdot V_{1}-\sum_{i=1}^{n} q_{j i} v_{j}^{\prime}
$$

or in vector form as earlier

$$
d^{2} \varphi\left(\mathbf{v}, U_{1}\right)=A_{\varphi \mathbf{v}} \cdot V_{1}-Q_{\varphi \mathbf{v}}^{\mathrm{T}} \cdot \mathbf{v}^{\prime}
$$

where $d^{2} \varphi\left(\mathbf{v}, U_{1}\right)$ is a column vector with $i$ th entry the vector $d^{2} \varphi_{x_{0}}\left(v_{i}, U_{1}\right)$. Then, $Q_{\varphi \mathbf{v}}=\left(q_{i j}\right)$ is a matrix representation of $Q_{\varphi}$ with respect to the basis $\mathbf{v}$.

Likewise at a point $x_{0} \in \partial M$, we define the edge distortion operator $Q_{\mathrm{E} \varphi}$ by

$$
Q_{\mathrm{E} \varphi}(v)=-d \varphi^{-1}\left(\operatorname{proj}_{V}^{\prime}\left(d^{2} \varphi_{x_{0}}\left(v, U_{1}\right)\right)\right) .
$$

We let $\left\{v_{1}, \ldots, v_{n}\right\}$ be a special basis for $T_{x_{0}} M$ so $\left\{v_{1}, \ldots, v_{n-1}\right\}$ is a basis for $T_{x_{0}} \partial M$ and $v_{n}$ maps under the edge parametrization to $c \cdot U_{1}$. Again we write $v_{i}^{\prime}=d \varphi\left(v_{i}\right)$, and let $\mathbf{n}^{\prime}$ be a unit normal vector field to $M^{\prime}$ in a neighborhood of the edge point $x_{0}^{\prime}=\varphi\left(x_{0}\right)$. Then, we analogously obtain, for $i=1, \ldots, n$,

$$
d^{2} \varphi_{x_{0}}\left(v_{i}, U_{1}\right)=a_{\varphi i} \cdot V_{1}-c_{\varphi i} \cdot \mathbf{n}^{\prime}-\sum_{j=1}^{n-1} b_{\varphi j i} v_{j}^{\prime},
$$

or in vector form (for $n$-dimensional vectors)

$$
d^{2} \varphi\left(\mathbf{v}, U_{1}\right)=A_{\varphi \mathbf{v}} \cdot V_{1}-C_{\varphi \mathbf{v}} \cdot \mathbf{n}^{\prime}-B_{\varphi \mathbf{v}} \cdot \tilde{\mathbf{v}}^{\prime}
$$

$\left(\tilde{\mathbf{v}}^{\prime}\right.$ denotes the $n-1$ dimensional column vector with $i$ th entry $\left.v_{i}\right)$. We let $Q_{\mathrm{E} \varphi, \mathbf{v}}=\left(\begin{array}{ll}B_{\varphi \mathbf{v}} & C_{\varphi \mathbf{v}}\end{array}\right)^{\mathrm{T}}$. Then $Q_{\mathrm{E} \varphi, \mathbf{v}}$ is the matrix representation for $Q_{\mathrm{E} \varphi}$ with respect to the special basis $\left\{v_{1}, \ldots, v_{n}\right\}$ in the source and $\left\{v_{1}, \ldots, v_{n-1}, \mathbf{n}\right\}$ in the target.

Third, because $\varphi$ need not preserve orthogonality to the skeletal set at the edge points, we also express

$$
d \varphi_{x_{0}}(\mathbf{n})=a_{\mathbf{n}} \cdot V_{1}+c_{\mathbf{n}} \cdot \mathbf{n}^{\prime}+\sum_{j=1}^{n-1} b_{\mathbf{n} j} \cdot v_{j}^{\prime} .
$$

Then, we define the $n \times n$ matrix $E_{\varphi \mathbf{v}}$ whose $(j, i)$ entry is $e_{j i}=c_{i} \cdot b_{\mathbf{n} j}$ for $j<n$ and $e_{n i}=c_{i} \cdot\left(c_{\mathbf{n}}-1\right)$, with $c_{i}$ denoting the $i$ th entry of $C_{U}$ in (1.4).

\section{Radial and edge curvature conditions for the image skeletal structure}

We next see that $Q_{\varphi \mathbf{v}}$ measures how $\varphi$ contributes to the radial curvature of the image $\left(M^{\prime}, V\right)$. While $Q_{\mathrm{E} \varphi, \mathbf{v}}$ also measures the contribution of $\varphi$ to the edge shape operator of $\partial M^{\prime}$ at edge points, there is a second contribution from $E_{\varphi \mathbf{v}}$, resulting from the failure of $\varphi$ to preserve orthogonality to the skeletal set on the edge points.

We relate the radial and edge shape operators for the two skeletal structures as follows. 


\section{J. DAMON}

THEOREM 5.4. With the above notation, we obtain the following:

1) For a non-edge point $x_{0} \in M$ and choice of smooth value for $U$ (on a local component of $x_{0}$ ), with corresponding value for $V$, let $S_{\mathbf{v}}$, respectively $S_{\mathbf{v}^{\prime}}$, denote the matrix representations of the radial shape operators at $x_{0}$ for the basis $\left\{v_{1}, \ldots, v_{n}\right\}$, respectively $x_{0}^{\prime}=\varphi\left(x_{0}\right)$ for the basis $\left\{v_{1}^{\prime}, \ldots, v_{n}^{\prime}\right\}$. Then

$$
S_{\mathbf{v}^{\prime}}=\sigma\left(S_{\mathbf{v}}+Q_{\varphi \mathbf{v}}\right) .
$$

2) For a point $x_{0} \in \partial M$, let $S_{\mathrm{E} \mathbf{v}}$, respectively $S_{\mathrm{Ev}^{\prime}}$, denote the matrix representations of the edge shape operators at $x_{0}$ for the special basis $\left\{v_{1}, \ldots, v_{n}\right\}$, respectively $x_{0}^{\prime}=\varphi\left(x_{0}\right)$ for the special basis $\left\{v_{1}^{\prime}, \ldots, v_{n}^{\prime}\right\}$. Then,

$$
S_{\mathrm{E} \mathbf{v}^{\prime}}=\sigma\left(S_{\mathrm{E} \mathbf{v}}+Q_{\mathrm{E} \varphi, \mathbf{v}}+E_{\varphi \mathbf{v}}\right)
$$

Remark 5.5. If $d \varphi$ preserves orthogonality to the skeletal set at the point $x_{0} \in \partial M$ in the sense that $d \varphi\left(x_{0}\right)(\mathbf{n})=\mathbf{n}^{\prime}$, then at $x_{0}, c_{\mathbf{n}}=1$ and $b_{\mathbf{n} i}=0$ for $1 \leqslant i \leqslant n-1$, and so $E_{\varphi \mathbf{v}}=0$. Then, the edge shape operator is only altered by the effects of $d^{2} \varphi$.

Proof of Theorem 5.4. For part 1, we must compute $\partial V_{1} / \partial v_{i}^{\prime}$ at a point of $x_{0} \in M_{\text {reg. Then, }}$.

$$
\begin{aligned}
\frac{\partial V_{1}}{\partial v_{i}^{\prime}} & =\frac{\partial}{\partial v_{i}^{\prime}}\left(\sigma \circ \varphi^{-1} \cdot d \varphi\left(U_{1}\right) \circ \varphi^{-1}\right) \\
& =\left(\frac{\partial \sigma}{\partial v_{i}} \circ \varphi^{-1}\right) \cdot d \varphi\left(U_{1}\right) \circ \varphi^{-1}+\left(\sigma \circ \varphi^{-1}\right) \cdot \frac{\partial\left(d \varphi\left(U_{1}\right)\right)}{\partial v_{i}} \circ \varphi^{-1} .
\end{aligned}
$$

After differentiating, we will not include composition with $\varphi^{-1}$ which will be understood. We note that the derivative in the second term on the right-hand side of $(5.12)$ is really $\partial\left(d \varphi_{x}\left(U_{1}(x)\right)\right) / \partial v_{i}$, so we compute it by applying the chain rule:

$$
\frac{\partial}{\partial v_{i}}\left(d \varphi_{x}\left(U_{1}(x)\right)\right)=d^{2} \varphi_{x}\left(v_{i}, U_{1}\right)+d \varphi_{x}\left(\frac{\partial U_{1}}{\partial v_{i}}\right) .
$$

Then, by (5.12), (5.13), (5.4), and (1.2) we obtain

$$
\begin{aligned}
\frac{\partial V_{1}}{\partial v_{i}^{\prime}} & =\frac{\partial \sigma}{\partial v_{i}} d \varphi\left(U_{1}\right)+\sigma\left(a_{\varphi i} \cdot V_{1}-\sum_{j=1}^{n} q_{j i} v_{j}^{\prime}\right)+\sigma \cdot d \varphi_{x}\left(a_{i} \cdot U_{1}-\sum_{j=1}^{n} s_{j i} v_{j}\right) \\
& =\left(\frac{\partial \sigma}{\partial v_{i}} \cdot \sigma^{-1}+\sigma \cdot a_{\varphi i}+a_{i}\right) \cdot V_{1}-\sigma\left(\sum_{j=1}^{n}\left(s_{j i}+q_{j i}\right) v_{j}^{\prime}\right) .
\end{aligned}
$$

We can write this in vector form as

$$
\frac{\partial V_{1}}{\partial \mathbf{v}^{\prime}}=\tilde{A}_{\varphi \mathbf{v}^{\prime}} \cdot V_{1}-\sigma\left(S_{\mathbf{v}}+Q_{\mathbf{v}}\right)^{\mathrm{T}} \cdot \mathbf{v}^{\prime}
$$

where

$$
\tilde{A}_{\varphi \mathbf{v}^{\prime}}=\sigma^{-1} \cdot d \sigma(\mathbf{v})+\sigma \cdot A_{\varphi \mathbf{v}^{\prime}}+A_{\mathbf{v}} .
$$

Applying $\operatorname{proj}_{V}$ and then $-d \varphi$ to Equation (5.15) yields (5.10).

For part 2, we analogously compute $\partial V_{1} / \partial v_{i}^{\prime}$ at a point $x_{0} \in \partial M$, using instead (5.7), (5.9), and (1.4). We obtain in vector form

$$
\frac{\partial V_{1}}{\partial \mathbf{v}^{\prime}}=A_{V \mathbf{v}^{\prime}} \cdot V_{1}-\sigma\left(S_{\mathrm{E} \mathbf{v}}+Q_{\mathrm{E} \varphi, \mathbf{v}}+E_{\varphi \mathbf{v}}\right)^{\mathrm{T}} \cdot\left(\begin{array}{c}
\tilde{\mathbf{v}}^{\prime} \\
\mathbf{n}^{\prime}
\end{array}\right),
$$

where $\tilde{\mathbf{v}}^{\prime}$ is the $(n-1)$-dimensional column vector with $i$ th entry $v_{i}^{\prime}$ and where the actual form of $A_{V \mathbf{v}^{\prime}}$ is not important. Again, applying $\operatorname{proj}_{V}^{\prime}$ and $-d \varphi$ to (5.16) yields (5.11). 


\section{GEOMETRY OF BOUNDARIES}

We let $\left\{b_{i}\right\}$ denote the eigenvalues of $S_{\mathbf{v}}+Q_{\varphi \mathbf{v}}$, and $\left\{d_{i}\right\}$ denote the generalized eigenvalues of $\left(S_{\mathrm{E} \mathbf{v}}+Q_{\mathrm{E} \varphi, \mathbf{v}}+E_{\varphi, \mathbf{v}}, I_{n-1,1}\right)$. Then, we note the following as a corollary.

Corollary 5.6. Let $\varphi:(M, U) \rightarrow\left(M^{\prime}, V\right)$ be a diffeomorphism of skeletal structures. Then, $\left(M^{\prime}, V\right)$ satisfies the radial curvature condition if and only if at all non-edge points of $M$

$$
r<\min \left\{\frac{1}{b_{i}}\right\} \quad \text { for all positive eigenvalues } b_{i} \text { of } S_{\mathbf{v}}+Q_{\varphi \mathbf{v}} .
$$

Likewise, $\left(M^{\prime}, V\right)$ satisfies the edge condition if and only if at all points of $\partial M$,

$r<\min \left\{\frac{1}{d_{i}}\right\} \quad$ for all positive generalized eigenvalues $d_{i}$ of $\left(S_{\mathrm{E} \mathbf{v}}+Q_{\mathrm{E} \varphi, \mathbf{v}}+E_{\varphi, \mathbf{v}}, I_{n-1,1}\right)$.

Proof. By Theorem 5.4, the principal radial curvatures are the eigenvalues of $\sigma\left(S_{\mathbf{v}}+Q_{\varphi \mathbf{v}}\right)$, i.e. $\sigma \cdot b_{i}$; and the principal edge curvatures are the generalized eigenvalues of $\sigma\left(S_{\mathrm{E} \mathbf{v}}+Q_{\mathrm{E} \varphi, \mathbf{v}}+E_{\varphi, \mathbf{v}}\right)$, i.e. $\sigma \cdot d_{i}$. Thus, the radial curvature condition requires

$$
\frac{r}{\sigma}=r_{1}<\min \left\{\frac{1}{\sigma \cdot b_{i}}\right\} \quad \text { for all positive eigenvalues } b_{i} .
$$

This is equivalent to (5.17). A similar argument establishes (5.18).

Thus, Corollary 5.6 provides us with concrete criteria for checking the smoothness of the associated boundary for $\left(M^{\prime}, V\right)$ away from singular points by only using the radial curvature and distortion data on the original $M$.

At singular points we combine the preceding results to obtain sufficient conditions in terms of the shape operators and distortion operators on $M$ in the case $\varphi$ is radially rigid. We must separately verify that the image skeletal structure satisfies the initial local conditions from part I [Dam03, Definition 1.7]. However, provided the image skeletal structure satisfies the initial local conditions, we can combine the results of Corollary 5.6 and Lemma 5.3 together with Theorem 2.3 of [Dam03] to conclude the smoothness of the boundary for an image skeletal structure.

Theorem 5.7. Suppose that $\varphi:(M, U) \rightarrow\left(M^{\prime}, V\right)$ is a diffeomorphism of skeletal structures which is radially rigid at the singular points of $M$. Suppose $M$ satisfies the compatibility condition on $M_{\text {sing }}$ and at non-edge points

$$
r<\min \left\{\frac{1}{b_{i}}\right\} \quad \text { for all positive eigenvalues } b_{i} \text { of } S_{\mathbf{v}}+Q_{\varphi \mathbf{v}}
$$

and at all points of $\overline{\partial M}$,

$$
r<\min \left\{\frac{1}{d_{i}}\right\} \quad \text { for all positive generalized eigenvalues } d_{i} \text { of }\left(S_{\mathrm{E} \mathbf{v}}+Q_{\mathrm{E} \varphi, \mathbf{v}}+E_{\varphi, \mathbf{v}}, I_{n-1,1}\right) .
$$

Provided $\left(M^{\prime}, V\right)$ satisfies the local initial conditions [Dam03, Definition 1.7], then it has a smooth boundary in the sense of Theorem 2.3 of Part I [Dam03].

\section{Geometry of the associated boundary of the image skeletal structure}

The results of Theorem 5.4 also allow us to compute the differential geometry of the associated boundary of the image skeletal structure $\left(M^{\prime}, V\right)$.

Corollary 5.8. Let $\varphi:(M, U) \rightarrow\left(M^{\prime}, V\right)$ be a diffeomorphism of skeletal structures which is radially rigid on an open set $Z \subset M$. Suppose $\left(M^{\prime}, V\right)$ satisfies the conditions (5.17) and (5.18) on $Z$, and that $x_{0} \in Z$ with a choice of smooth value of $U$ on a local component of $x_{0}$. 


\section{GEOMETRY OF BOUNDARIES}

1) If $x_{0}$ is not an edge closure point, then

$$
S_{\mathcal{B}^{\prime} \mathbf{v}^{\prime}}=\left(I-r\left(S_{\mathbf{v}}+Q_{\varphi \mathbf{v}}\right)\right)^{-1} \cdot \sigma\left(S_{\mathbf{v}}+Q_{\varphi \mathbf{v}}\right) .
$$

2) If $x_{0}$ is an edge closure point then

$$
S_{\mathcal{B}^{\prime} \mathbf{v}^{\prime}}=\left(I_{n-1,1}-r\left(S_{\mathrm{E} \mathbf{v}}+Q_{\mathrm{E} \varphi, \mathbf{v}}+E_{\varphi, \mathbf{v}}\right)\right)^{-1} \cdot \sigma\left(S_{\mathrm{E} \mathbf{v}}+Q_{\mathrm{E} \varphi, \mathbf{v}}+E_{\varphi, \mathbf{v}}\right) .
$$

Remark 5.9. With the assumptions of Corollary 5.8, if both $S_{\mathcal{B} \mathbf{v}}$ and $S_{\mathcal{B}^{\prime} \mathbf{v}^{\prime}}$ are invertible, we may use (5.19) to rewrite (3.7) as follows:

$$
\sigma^{-1} S_{\mathcal{B}^{\prime} \mathbf{v}^{\prime}}^{-1}=S_{\mathcal{B} \mathbf{v}}^{-1}-\left(S_{\mathbf{v}}^{-1} Q_{\varphi, \mathbf{v}}\left(S_{\mathbf{v}}+Q_{\varphi, \mathbf{v}}\right)^{-1}\right) .
$$

We see that (5.21) is a consequence of (3.7) by using (5.19) and $r_{1} \circ \varphi=r$ to write

$$
\sigma^{-1} S_{\mathcal{B}^{\prime} \mathbf{v}^{\prime}}^{-1}-S_{\mathcal{B} \mathbf{v}}^{-1}=\left(S_{\mathbf{v}}+Q_{\varphi, \mathbf{v}}\right)^{-1}-S_{\mathbf{v}}^{-1}
$$

which we easily verify implies (5.21).

\section{REFERENCES}

BN78 H. Blum and R. Nagel, Shape description using weighted symmetric axis features, Pattern Recognition 10 (1978), 167-180.

BGT96 J. W. Bruce, P. J. Giblin and F. Tari, Ridges, crests, and subparabolic lines of evolving surfaces, Int. J. Comp. Vision 18 (1996), 195-210.

Dam03 J. Damon, Smoothness and geometry of boundaries associated to skeletal structures I: Sufficient conditions for smoothness, Ann. Inst. Fourier (Grenoble) 53 (2003), 1941-1985.

Dam04 J. Damon, Determining the geometry of boundaries of objects from medial data, Int. J. Comp. Vision, to appear.

Gib00 P. J. Giblin, Symmetry sets and medial axes in two and three dimensions, in The Mathematics of Surfaces, eds R. Cipolla and R. Martin (Springer, Berlin, 2000), 306-321.

Nac82 L. R. Nackman, Three-dimensional shape description using the symmetric axis transform, $\mathrm{PhD}$ thesis, University of North Carolina (1982).

NP85 L. R. Nackman and S. Pizer, Three-dimensional shape description using the symmetric axis transform I: Theory, IEEE Trans. Pattern Anal. Machine Intell. 7 (1985), 187-202.

Sie99 D. Siersma, Properties of conflict sets in the plane, in Geometry and Topology of Caustics - Caustics 1998, Banach Center Publ. 50 (1999), 267-276.

SSG99 J. Sotomayor, D. Siersma and R. Garcia, Curvatures of conflict surfaces in Euclidean 3-space, in Geometry and Topology of Caustics - Caustics 1998, Banach Center Publ. 50 (1999), 277-285.

Spi75 M. Spivak, A comprehensive introduction to differential geometry, vols I-V (Publish or Perish, Berkeley, CA, 1975, 1979).

VaM03a M. Van Manen, Curvature and torsion formulas for conflict sets, in Caustics '02 (Warsaw) (Polish Academy of Sciences, Warsaw, 2003).

VaM03b M. Van Manen, The geometry of conflict sets, PhD Thesis, University of Utrecht (2003).

James Damon jndamon@math.unc.edu

Department of Mathematics, University of North Carolina, Chapel Hill, NC 27599-3250, USA 